\title{
Aplicación del Modelo de Aceptación Tecnológica en Sistemas de Información de la Administración Pública del Perú
}

\section{Application of the Technology Acceptance Model in Information Systems at the Public Administration of Peru}

\author{
Marlene Reyes 1,a, Pedro Castañeda 1,b \\ ${ }^{1}$ Universidad Nacional Mayor de San Marcos, Facultad de Ingeniería de Sistemas e Informática. Lima, Perú \\ a Email: areyesh@unmsm.edu.pe \\ ${ }^{\text {b} E m a i l: ~ p e d r o . c a s t a n e d a @ u n m s m . e d u . p e ~}$
}

\begin{abstract}
Resumen
El Modelo de Aceptación Tecnológica (TAM) es un modelo que es utilizado por muchos investigadores con el fin de analizar la aceptación tecnológica que permita garantizar el éxito de su implementación. El modelo TAM ha logrado posicionarse como uno de los modelos más utilizados, para probar y validar entre los modelos existentes para la aceptación tecnológica. El principal objetivo de esta investigación es analizar la adopción del Sistema de Gestión Documental (SGD) que utiliza tecnología de Firma Digital en las entidades públicas peruanas, incorporando al modelo TAM dos constructos importantes: la confianza y el riesgo percibido. Los diversos estudios demuestran que la capacidad predictiva de estos constructos psicológicos influye de manera positiva en concordancia con los constructos del modelo TAM. Las conclusiones de la presente investigación pueden permitir mejorar el servicio al ciudadano, el cual aportará de manera positiva a las decisiones de los gestores de la administración pública, académicos, docentes e investigadores en este entorno.
\end{abstract}

Palabras clave: TAM; riesgo percibido; confianza; Sistema de Gestión Documental.

\begin{abstract}
Technology Acceptance Model (TAM) is one model that is used by many researchers in order to analyze the technological acceptance that guarantees the success of its implementation. The TAM model has managed to position itself as one of the most used models, to test and validate among existing models for technological acceptance. The main objective of this research is to analyze the adoption of the Document Management System (SGD) that uses Digital Signature technology in Peruvian public entities, incorporating two important constructs into the TAM model: trust and perceived risk. The various studies show that the predictive capacity of these psychological constructs influences in a positive way in agreement with the constructs of the TAM model. The conclusions of the present investigation can improve the service to citizens, which will contribute positively to the decisions of public administration managers, academics, teachers and researchers in this environment.
\end{abstract}

Keywords: TAM; perceived risk; confidence; Document Management System.

Correspondencia:

Dirección: Universidad Nacional Mayor de San Marcos, Facultad de Ingeniería de Sistemas e Informática. Calle Germán Amézaga N³75, Ciudad Lima 1.

Recibido 13/10/2019 - Aceptado 25/06/2020

Citar como:

Reyes, M. \& Castañeda, P. (2020) Aplicación del Modelo de Aceptación Tecnológica en Sistemas de Información de la Administración Pública del Perú. Revista Peruana de Computación y Sistemas, 3(1):15-22. http://dx.doi.org/10.15381/rpcs.v3i1.18350

(C) Los autores. Este artículo es publicado por la Revista Peruana de Computación y Sistemas de la Facultad de Ingeniería de Sistemas e Informáticade la Universidad Nacional Mayor de San Marcos. Este es un artículo de acceso abierto, distribuido bajo los términos de la licencia Creative Commons Atribucion - No Comercia_Compartir Igual 4.0 Internacional. (http://creativecommons.org/licenses/by-nc-sa/4.0/) que permite el uso no comercial, distribución y reproducción en cualquier medio, siempre que la obra original sea debidamente citada. 


\section{Introducción}

La adopción y uso de las tecnologías de la información y comunicaciones (TIC), se viene incrementando en la Administración Pública, fomentando políticas de internet en los gobiernos [1], por lo cual utilizan una gran variedad de sistemas y herramientas tecnológicas, que buscan brindar un servicio de calidad al ciudadano.

El Banco Interamericano de Desarrollo, en su informe denominado "el fin del trámite eterno", menciona que actualmente, en América Latina y el Caribe, el $89 \%$ de los trámites administrativos se realiza de forma presencial [2], siendo más costosos respecto a los trámites virtuales. La demanda del uso de las TIC por parte de los ciudadanos está motivando a la administración pública a la incorporación de servicios en línea, cuyo rol se ha vuelto más dinámico [1].

Adoptar una tecnología implica su máximo aprovechamiento (uso), considerando además que la facilidad de uso y la utilidad percibida de una tecnología pueden afectarla de manera positiva o negativa [3].

Las actividades manuales, la necesidad de la presencia física del ciudadano y la falta de estandarización de los procesos hacen que los trámites sean una vulnerabilidad para actos deshonestos [2], evidenciándose que un $21 \%$ de los latinoamericanos hizo una denuncia por haber realizado un pago de soborno dentro del contexto de un servicio público [4].

En América Latina y el Caribe, los gastos que ocasionan los trámites que se realizan en forma presencial son mayores respecto a los de manera virtual, necesitando de tres a más interacciones para resolver un trámite, tal es el caso de Bolivia, seguido de Perú, Guatemala, Panamá, Brasil, Venezuela, México y Colombia por encima del promedio alto [2].

Sin embargo, empoderar a los ciudadanos y aprovechar el poder de las nuevas tecnologías, se viene realizando en el Perú desde el año 2012, en la que forma parte de la Alianza para el Gobierno Abierto, que busca que los gobiernos sean más eficaces y responsables con la "Declaración de Gobierno Abierto", respaldado por 75 países [5].

A ello se suma el Decreto Legislativo $\mathrm{N}^{\circ} 1412$, que aprueba la Ley de Gobierno Digital, que tiene como finalidad mejorar la prestación de servicios digitales mediante la interoperabilidad entre las entidades públicas y promoviendo la transparencia y participación del ciudadano, buscando generar servicios con valor público a través del uso estratégico de las tecnologías digitales [6].

En el Perú se tiene como objetivo la implementación del Sistema de Gestión Documental (SGD) con tecnología de Firma Digital en las entidades del Poder Ejecutivo, establecido como parte del Modelo de Gestión Documental y en la que establecen fechas de implementación, en un primer momento a diciembre del 2018 [7], en un segundo momento a diciembre del
2019 [8], sin embargo, mediante Decreto de Urgencia No 006-2020 el plazo ha sido extendido hasta el 31 de diciembre de 2021. Si bien, existen plazos para la implementación, no existen investigaciones previas que analicen el comportamiento y la aceptación del SGD por el usuario de la administración pública peruana.

Según el Portal de Datos Abiertos de la Presidencia de Consejo de Ministros, en el Perú existen 2940 entidades públicas del estado peruano [9], de las cuales solo 22 entidades han implementado el sistema de información para Trámite Documentario con Firma Digital [10], equivalente a un $0.007 \%$ del total de entidades públicas, estando aún pendiente la implementación en un $99.9 \%$ [10].

Las soluciones tecnológicas que se promueven en la administración pública, como es el caso del SGD, son sistemas de gran impacto y de gran escala, motivo por el cual la presente investigación se centra en validar el uso y comportamiento de los usuarios de la administración pública peruana con el SGD, mediante el grado de aceptación y uso de la misma, tomando como referencia el modelo TAM e incorporando dos variables externas (confianza y riesgo percibido) [11], los cuales son fundamentales cuando se usan sistemas de entornos web, como es el caso del SGD, las redes sociales virtuales, TIC, e-learning, aplicativos móviles financieros, efectivo móvil, comercio electrónico, redes sociales en formación, apps móviles ([11],[12],[13],[14], $[15],[16],[17],[18])$. Sin embargo, cabe precisar que el SGD además de ser un sistema de entorno web, incorpora el uso de certificados y firmas digitales, por lo que el riesgo percibido y la confianza son factores latentes necesarios de analizar y evaluar.

Entre los modelos más utilizados para dicho análisis se encuentra el modelo TAM, por lo que muchos estudios han validado el TAM y sus variantes, además el TAM ha sido referenciado y citado, más de 95,000 veces desde su publicación según Google Scholar, haciendo referencia a los dos paper propuesto por Fred Davis, MIS Quarterly y el paper in Management Science have been the subject [19]. El TAM sigue siendo hasta la actualidad el modelo con mayor reputación para explicar la aceptación y adopción de una tecnología [13]. Asimismo, Gutarra [20] precisa que: "El TAM es un instrumento confiable y válido para predecir la adopción de tecnología; por lo que el TAM con sus postulados se aplica en el Perú y puede trabajarse en otros contextos para evaluar diversos problemas de adopción de tecnología en distintos ámbitos" (párr. 7).

La presente investigación está centrada en una población peruana y con una herramienta tecnológica nueva, cuyo desafío es analizar su uso y comportamiento en la administración pública, que no ha sido probada hasta el momento.

Este trabajo está dividido en siete secciones. La primera, realiza una introducción, segundo se realiza la revisión de la literatura. En la tercera sección se realiza 
el planteamiento de las hipótesis de investigación, posteriormente se detalla el modelo propuesto. En la quinta sección se procede con la metodología de la investigación, validando el modelo planteado y la contrastación de hipótesis. La sexta sección muestra los resultados y finalmente en la séptima sección se presentan las conclusiones de la investigación.

\section{Revisión de Literatura}

\subsection{Modelo de Aceptación Tecnológica (TAM)}

TAM tiene sus inicios en el modelo conceptual de Davis en la que sostiene, que el uso actual de la tecnología es directamente influenciado por la motivación del usuario para utilizar el sistema y que a la vez está directamente relacionada por un estímulo externo con características y capacidades del sistema [21].

Los constructos del TAM están formados por la actitud, la utilidad percibida, la facilidad de uso, intención hacia el uso y el uso [21], [22].

La siguiente versión del TAM fue realizada por Davis dos años después [23], en la que demuestra que la utilidad percibida y la facilidad de uso percibida influyen de manera directa en la intención de uso, eliminándose el constructo actitud hacia el uso, proponiendo la nueva versión del TAM.

Si bien la actitud y la intención conductual o intención de uso quedan definidos en el TAM ([22],[24],[25]), la utilidad percibida se conceptualiza como el grado de creencia del usuario acerca de la contribución de un sistema a su desempeño en el trabajo ([26]), mientras que la facilidad de uso percibida, es el grado en el que una persona espera que el sistema no presente dificultad en el uso ([19],[26]). De acuerdo a Valera et al. [27], TAM ha sido utilizado con éxito en muchas investigaciones de campo.

Según Valera et al. [27], las variables externas que impactan en la utilidad y la facilidad de uso percibida determinan la relación entre ellas, predicen la aceptación tecnológica y si esta será utilizada de manera óptima.

El modelo TAM ha sido aplicado en diferentes tecnologías como en redes sociales virtuales, recompra online, software de gestión, correos electrónicos, plataforma Nexus, reconocimiento facial, tecnologías móviles, aplicativos móviles financieros, sistemas de información. ([12],[28],[29],[30],[31],[32],[33], [34]).

A continuación, en la Tabla 1, se muestra estudios realizados aplicando el modelo TAM a los diferentes tipos de herramientas tecnológicas de entorno web incorporando el constructo riesgo percibido y/o confianza, como es el caso del SGD.

De acuerdo a lo mostrado en la Tabla 1 , no existen investigaciones previas que analicen el comportamiento y la aceptación de tecnologías por parte del usuario de la administración pública peruana.

Cabero et al. [40], señalan que: "aunque el modelo TAM ha ido evolucionando ([41], [42]) sigue estando constituido en su núcleo por un conjunto simple de variables identificadas en la primera formulación, lo que es posiblemente su gran ventaja en la aplicación, y lo hace válido y robusto”.

\subsection{El Sistema de Gestión Documental (SGD) con tecnología de firma digital en la Administración Pública Peruana}

El SGD, es una herramienta tecnológica web, que incorpora el uso de los Certificados Digitales, con el objetivo de gestionar los documentos (internos y externos) de la administración pública peruana. El SGD tiene incorporado el software de firma acreditado, llamado firma ONPE, que permite firmar de manera digital diferentes tipos de documentos en formato PDF. Cuenta con un módulo administrador, un módulo de gestor y control de accesos, que permite generar vistos buenos múltiples y anexar diferentes tipos de archivos, como videos, audios, imágenes, hojas de cálculo, etc. [43].

Con la aprobación del Modelo de Gestión Documental en el marco del Decreto Legislativo $\mathrm{N}^{\circ} 1310$, de fecha 9 de agosto de 2017, se establece que las entidades del Poder Ejecutivo deben incorporar el uso de Herramientas Informáticas, certificados y firmas digitales para la gestión documentaria [7].

Tabla 1. Estudios realizados en base al Modelo de Aceptación Tecnológica (TAM) de entorno web

\begin{tabular}{llll} 
Tecnología & Modelo & País & Fuente \\
Software de Gestión de Proyectos & TAM Adaptado & Perú & {$[29]$} \\
Correo Electrónico & TAM/UTAUT & España & {$[30]$} \\
Reconocimiento Facial & TAM/TRI & Chile & {$[32]$} \\
e-Commerce & TAM Adaptado/TPB & Colombia & {$[16]$} \\
e-Gobierno & TAM/UTAUT & España & {$[35]$} \\
Redes Sociales Virtuales & TAM Adaptado & Perú & {$[36]$} \\
Comercio Electrónico & UTAUT, TAM y TRA & Varios & {$[37]$} \\
e-Commerce & TAM/TPB & Colombia & {$[38]$} \\
Sitios Web de Compras & TAM Adaptado & Colombia & {$[39]$} \\
\hline
\end{tabular}


Según el Portal de Datos Abiertos de la Presidencia de Consejo de Ministros, se tiene pendiente la implementación del SGD en un $99.9 \%$ [10].

En el contexto peruano no existen investigaciones que permitan predecir la aceptación del SGD con Tecnología de Firma Digital, mediante el uso del modelo TAM considerando las variables confianza y riesgo percibido.

\section{Hipótesis}

Se propone las siguientes hipótesis para determinar la influencia en el uso del SGD de los constructos: Actitud hacía el Uso (AHU), Intención Hacía el Uso (IHU), Utilidad Percibida (UP), Facilidad de Uso Percibida (FUP), Riesgo Percibido (RP) y Confianza (C).

\section{Influencia del constructo Actitud Hacia el Uso (AHU)}

TAM comprende la actitud hacia el uso proveniente de la TRA (Theory of Reasoned Action) [22] en la que Davis [21] afirma que la actitud hacia el uso como un antecedente de la intención de uso, la cual depende de las variables: utilidad percibida y facilidad de uso percibida. Así lo demuestran los estudios que aplican el TAM ([11],[28],[17], [44]).

H1: La Actitud Hacia el Uso del SGD influye positivamente en la Intención Hacia el Uso del sistema.

\section{Influencia del constructo Intención Hacía el Uso (IHU)}

Según los modelos TAM [21],[25] incorporan la variable intención de uso, el cual tiene un efecto directo en la utilidad percibida y la actitud hacia el uso, que a su vez determina el comportamiento para el uso tecnológico [21],[25] y que es útil para explicar la aceptación tecnológica por ejemplo de sitios web [39]. Por tanto, en esta línea proponemos la siguiente hipótesis:

H2: La Intención Hacia el Uso del SGD influye positivamente en el Uso del Sistema.

\section{Influencia del constructo Utilidad Percibida (UP)}

Davis [21] afirma que la utilidad percibida influye de manera directa en el uso a través de la intención de uso. Asimismo, diversos estudios sustentan la relación directa entre la utilidad y la intención en los modelos TAM [28], [38],[34],[17].

H3: La Utilidad Percibida del SGD influye positivamente en la Intención Hacia el Uso del sistema.

La facilidad de uso percibido y la utilidad percibida determinan el uso de una tecnología influenciada por la actitud hacia el uso [21].

H4: La Utilidad Percibida del SGD influye positivamente en la Actitud Hacia el Uso del sistema.

Influencia del constructo Facilidad de Uso Percibida (FUP)
En base a la literatura [45], [46] se menciona que en el Modelo TAM la utilidad percibida está condicionada por la facilidad de uso percibida.

H5: La Facilidad de Uso Percibida del SGD influye positivamente en la Utilidad Percibida del sistema.

En TAM, la actitud hacia el uso de un sistema de información se basa en las variables: utilidad percibida y facilidad de uso percibida. De acuerdo a Muñoz [45], la facilidad de uso percibida está influenciada por la autoeficacia y la instrumentalidad, es por ello que este efecto de facilidad de uso está directamente relacionado con la actitud.

H6: La Facilidad de Uso Percibida del SGD influye positivamente en la Actitud Hacía el Uso del sistema.

En [21] se establece una relación indirecta entre la facilidad de uso percibida y la intención hacia el uso, interviniendo la utilidad percibida.

H7: La Facilidad de Uso Percibida del SGD influye positivamente en el Riesgo Percibido del sistema.

\section{Influencia del constructo Confianza (C)}

Diversos estudios ([11], [16], [28], [39], [47]) consideran a la confianza como un constructo determinante en la adopción o aceptación de las tecnologías, mencionando que mientras exista una mayor confianza de los usuarios hacia un sitio web, menor será el esfuerzo a considerar en los detalles del sitio. Asimismo, concluyen que la confianza influye en la utilidad percibida y en la facilidad de uso, generando que el usuario observe sus beneficios y genere confianza en su uso.

La confianza es un determinante indirecto de la intención de uso, y ejerce un efecto directo a través de la actitud [11].

H8: La Confianza hacia el SGD influye positivamente en la Utilidad Percibida del sistema

H9: La Confianza hacia el SGD influye positivamente en la Actitud Hacía el Uso del sistema.

H10: La Confianza hacia el SGD influye positivamente en la Facilidad de Uso Percibida del sistema.

\section{Influencia del constructo Riesgo Percibido (RP)}

La medida del riesgo percibido es un constructo a considerar en el modelo, ya que los usuarios, de manera consciente o inconsciente, tienen una percepción de riesgo cuando realizan la evaluación para la adopción y/o uso de tecnologías web, como es el caso del SGD. De acuerdo a investigaciones realizadas, el constructo riesgo percibido, surge como una combinación de dos componentes: la probabilidad de incurrir en una pérdida como consecuencia de una conducta y la importancia atribuida a dicha pérdida. El riesgo puede asociarse al soporte del sistema como tal, a la pérdida de información, al desconocimiento de la tecnología, 
a la posible clonación de su identidad, desconocimiento sobre cifrado, desconocimiento sobre firma y certificado digital, desconocimiento de jergas técnicas de seguridad de la información o la falta de comprensión [48],[49].

En la investigación realizada por Lorenzo Romero [11] indica que si el usuario logra percibir el riesgo tendría como consecuencia que su uso sea menor, sin embargo, en el caso que el usuario considera cómo fácil de usar el riesgo percibido será menor.

H11: El Riesgo Percibido del SGD influye positivamente en la Utilidad Percibida del sistema.

H12: El Riesgo Percibido del SGD influye positivamente en la Intención Hacia el Uso del sistema.

En la Tabla 2 se presenta, las hipótesis planteadas:

Tabla 2. Resumen de hipótesis

\begin{tabular}{lccccccc} 
Constructos & IHU & UP & AHU & FUP & C & RP & USO \\
AHU & H1 & & & & & & \\
\hline IHU & & & & & & & H2 \\
\hline UP & H3 & & H4 & & & & \\
\hline FUP & & H5 & H6 & & & H7 & \\
\hline C & & H8 & H9 & H10 & & \\
\hline RP & F12 & F11 & & & & & \\
\hline
\end{tabular}

\section{Modelo Propuesto}

Tal como se ha explicado en los párrafos anteriores, la intención de uso es el factor determinante para la implementación de una nueva tecnología, y tomando en consideración la literatura, se propone en la Fig. 1 el modelo de relaciones causales que explican los constructos: Facilidad de Uso Percibida, Utilidad Per- cibida, Actitud Hacía el Uso, Intención Hacía el Uso, Riesgo Percibido y Confianza que influyen o predicen el uso del SGD como herramienta de apoyo en la gestión administrativa.

El modelo propuesto explica que la facilidad de uso percibida influye de manera positiva en la utilidad percibida que se tiene del sistema. También, cuanto más útil se perciba mayor será la actitud hacia el uso del sistema y por lo tanto aumentará la intención de uso, que conllevará a un mayor uso.

Respecto a los constructos confianza y riesgo percibido del sistema, se puede establecer que mientras más confianza pueda generarse, habrá una mayor actitud positiva y la percepción será en términos de utilidad y facilidad de uso. Asimismo, si existiera una percepción de riesgo del sistema, el usuario las considerará de menos utilidad y ello conllevará a la disminución de la intención de uso. Por otra parte, si el usuario considera que el sistema es fácil de utilizar, la percepción de riesgo será menor y habrá una mayor expectativa en la intención de uso.

\section{Metodología}

Para la obtención de datos, se ha utilizado la técnica del muestreo, a fin de determinar la validez del modelo propuesto.

La muestra seleccionada consta de las siguientes actividades: (i) Utilización de fuente de información primaria (ii) Realización de la encuesta presencial a los usuarios del SGD de una entidad pública deportiva, con edades comprendidas entre 24 y 51 ańos de ambos sexos, ubicados en la ciudad de Lima Metropolitana. La encuesta se aplicó en el mes de marzo del 2018.

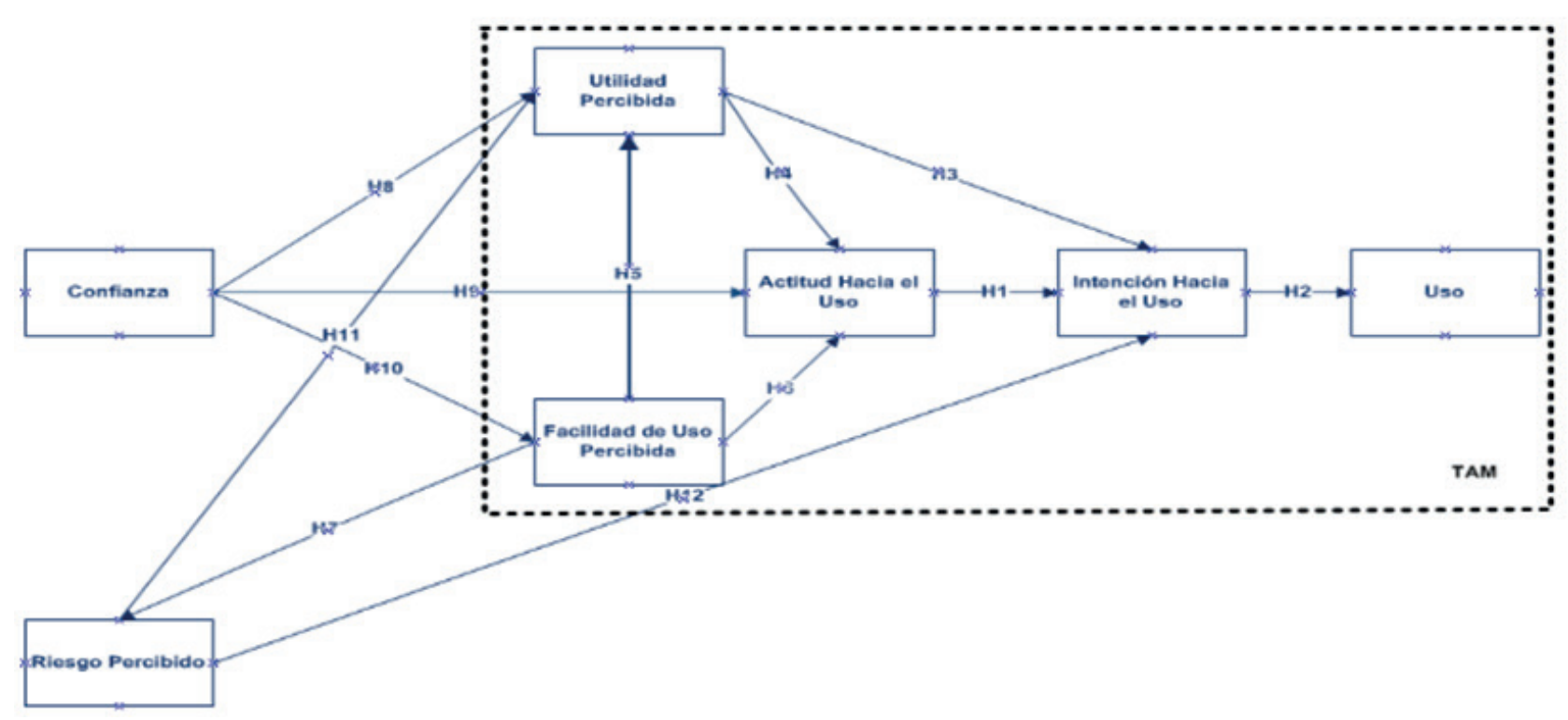

Fig. 1. Modelo propuesto 
El objetivo de la encuesta fue determinar el grado de aceptación y uso del SGD, tomando como referencia el modelo TAM. Para ello, utilizamos como instrumento de estudio el uso de cuestionarios basada en el modelo propuesto, empleando preguntas cerradas y de respuesta simple.

La encuesta se estructuró en 3 secciones: (i) Datos generales del usuario (2 preguntas simples), (ii) Uso del SGD (3 preguntas simples), (iii) Percepción y aceptación del SGD (32 preguntas matriciales) se evaluaron con la escala Likert de acuerdo con una calificación de 5 valores (1: totalmente en desacuerdo, 2: desacuerdo, 3: neutral, 4: de acuerdo, 5: totalmente de acuerdo).

Previamente a la encuesta, se realizó una prueba piloto para validar las preguntas del cuestionario, el cual fue realizado por 2 magister y un doctorado. El cuestionario se ajustó en la redacción.

La encuesta se realizó a 202 usuarios, descartando los cuestionarios que no fueron respondidas en su totalidad o los que tuvieron más de 2 respuesta por pregunta, obteniendo 171 encuestas válidas.

\section{Resultados}

Según Henseler [49], indica que para ajustar el modelo se puede hacer mediante índices de ajuste el cual proporciona valores aproximados. Para la presente investigación se realizó el análisis estadístico de ajuste del
SRMR (Standardized Root Mean Square Residual), CFI (Comparative Fix Index), y NFI (Normed Fit Index).

En general, se considera que el SRMR mide la diferencia entre la matriz de correlación observada y la matriz de correlaciones. Cuánto más bajo sea el SRMR tendrá mejor ajuste (SRMR <.1) [38].

El CFI debe estar cercano a 0.95 para que el modelo se ajuste de manera adecuada a los datos. Este valor, sin embargo, es relativo, pues, en modelos de gran complejidad el $\chi 2$ siempre se alejará de cero, lo que hace disminuir el CFI. En cuanto al NFI, cuánto más cerca de 1 , mejor.

Cabe señalar que de acuerdo a la Tabla 3, que el índice de chi-cuadrado $(\chi 2=1212.656)$ es estadísticamente significativo, así mismo, según la literatura, los índices para la SRMR (.089), CFI (.841) y NFI (.754), indicaron que el ajuste fue considerado adecuado para el modelo de investigación propuesto.

La figura 2, muestra el Modelo Factorial Recursivo, lo cual es de acuerdo a los niveles de aceptación requerido para validar el modelo.

Referente a la aplicación del modelo en el caso del SGD con tecnología digital, como herramienta de apoyo en la Administración Pública en el Perú, se han obtenido los siguientes resultados:

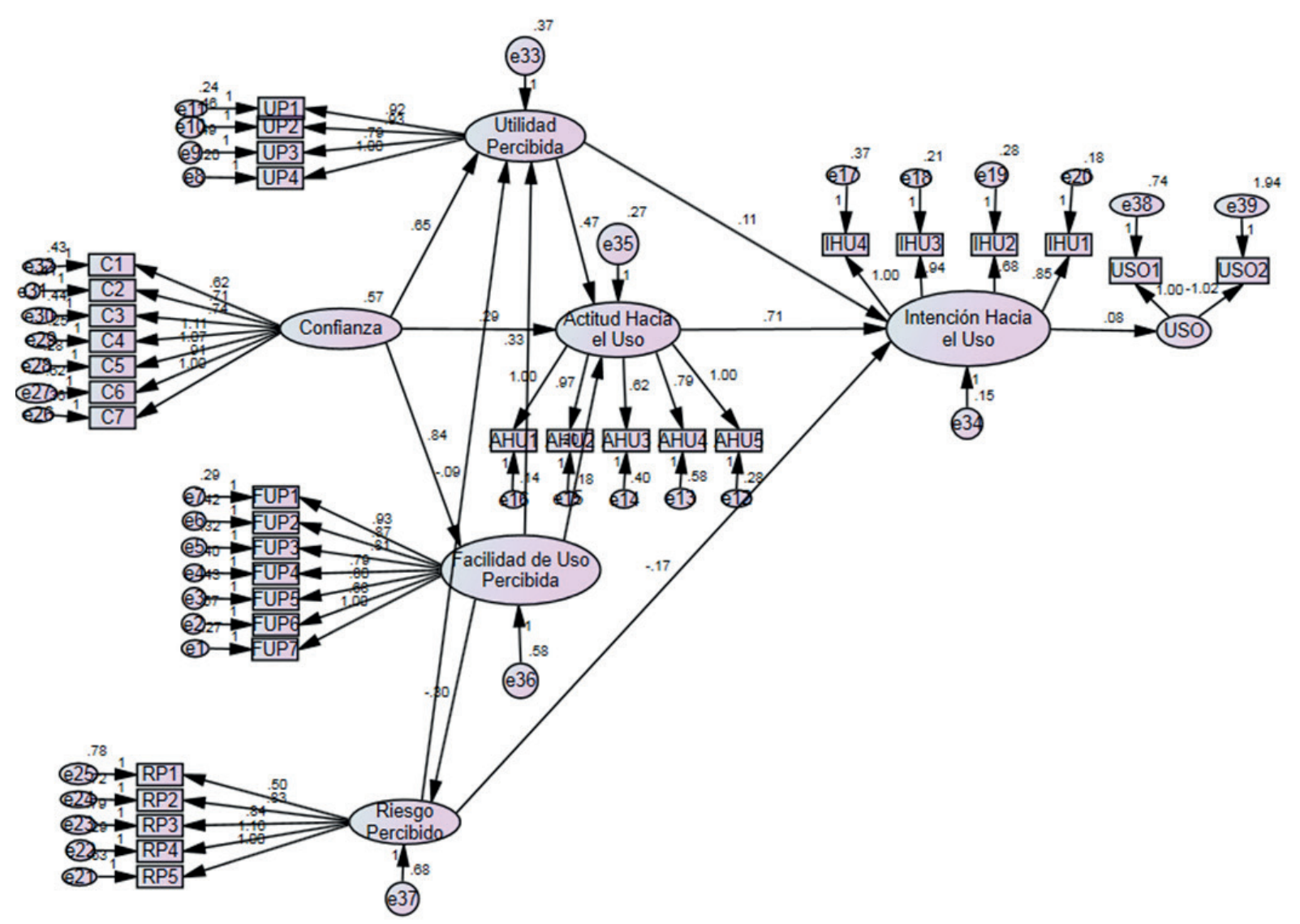

Fig. 2. Modelo estructural recursivo 


\section{Conclusiones}

La investigación se centra en analizar el uso, comportamiento y la adaptabilidad de los usuarios del SGD, analizando el grado de aceptación y uso de la misma, por lo cual queda demostrado según las hipótesis planteadas en la presente investigación, que el usuario del SGD se adapta positivamente al uso del SGD con certificado digital.

Por ello haciendo uso del modelo TAM ampliado, específicamente adicionando los constructos Riesgo Percibido y Confianza, el modelo planteado en la presente investigación explica que la facilidad de uso percibida del SGD con tecnología de certificado digital, como herramienta de apoyo en la Administración Pública en el Perú impacta positivamente en la utilidad percibida de esta herramienta. Asimismo, mientras que los usuarios tengan una mayor percepción de utilidad, mayor será la actitud positiva que se tenga hacia el SGD y por lo tanto aumentará la intención de uso, que conllevará a un mayor uso y adaptabilidad por parte de los usuarios.

Respecto a la confianza y el riesgo percibido del $S G D$, se concluye que cuanta más confianza genere el SGD se tendrá una actitud positiva y la percepción será de utilidad y facilidad de uso, por lo tanto, el usuario se adaptará fácilmente. Sin embargo, si hay una percepción de riesgo del SGD, el usuario las considerará menos útiles y su intención de uso será menor, generando menor expectativa en la intención de uso. Por otra parte, si el SGD es considerado por el usuario como fácil de usar, la percepción del riesgo se irá reduciendo, ya que los usuarios asumen que las actividades que son conocidas son menos arriesgadas, en consecuencia, conlleva a tener una mayor expectativa en la intención de usarla.

De lo anterior, si las instituciones públicas quieren masificar el uso de un Sistema de Gestión Documental en toda la administración pública, se debe considerar que sean usables y de utilidad para los usuarios del SGD, el cual permite que los perciban que el uso de esta herramienta tecnológica les facilitará el logro de sus objetivos y puedan adaptarse fácilmente.

La presente investigación es coherente con resultados de otras investigaciones utilizando el modelo TAM.

\section{Referencias}

[1] OCDE, Perspectivas de la OCDE sobre la economía digital 2017, México: OCDE, 2017. 3

[2] B. Roseth, A. Reyes y C. Santiso, Fin del trámite eterno: ciudadanos, burocracia y gobierno digital, New York: BID, 2018, p. 44.

[3] O. Y. Carrera Mora, S. A. Delgado de los Santos y M. C. Ovando Chico, «Factores que incentivan el uso de la biblioteca virtual en los estudiantes universitarios: un estudio de caso de la Universidad de Gómez Palacio de Durango,» Biblios, vol. 1, n 66, pp. 1-14, 2017.
[4] C. Pring, «Las personas y la corrupción: América Latina y el Caribe. Barómetro Global de Corrupción,» 2017. [En línea]. Available: https://www.transparency.org/whatwedo/publication/las_personas_y_la_corrupcion_america_latina_y_el_caribe.

[5] OGP. (2019). Open Goverment Partnership. Obtenido de https://www.opengovpartnership.org/process/joining-ogp/ open-government-declaration/

[6] El Peruano, Ley de Gobierno Digital-DL 1412, Lima: Editora Perú, 2018.

[7] Secretaria de Gobierno Digital, Modelo de Gestión Documental en el marco del Decreto Legislativo N 1310, Lima, Perú: El Peruano, 2017, pp. 4-37.

[8] El Peruano, Ley equilibrio financiero del Presupuesto del Sector Público para el año fiscal 2019, Lima: Editora Perú, 2019.

[8.a] El Peruano, Decreto de Urgencia N 006-2020, Lima: Editora Perú, 2020, pp. 3-6.

[9] PCM, «Datos Abiertos,» 2019. [En línea]. Available: https:// www.datosabiertos.gob.pe/dataset/lista-de-entidades-del-estado-peruano.

[10] Presidencia de Consejo de Ministro, «Cero Papel,» 13 Marzo 2018. Obtenido de: http://www.gobiernodigital.gob.pe/mgd/ Avance_Cero_Papel-Pod_Ejecutivo.xlsx

[11] Lorenzo Romero, Carlota; Alarcón de Amo, María del Carmen; Gómez Borja, Miguel Ángel; «Adopción de redes sociales virtuales: ampliación del modelo de aceptación de tecnológica integrando confianza y riesgo percibido,» Cuadernos de Economía y Dirección de la Empresa, vol. 14, n 1 , pp. 194-205, 2010.

[12] H. Guner y C. Acarturk, «The use and acceptance of ICT by senior citizens: a comparison of technology acceptance model (TAM) for elderly and young adults,» Universal Access in the Information Society, vol. 1, n 1, pp. 1-20, 2018.

[13] R. Arteaga Sánchez y A. Duarte Hueros, «Análisis de las plataformas de enseñanza virtuales,» vol. $1, n^{\circ} 1$, pp. 1-11, 2010.

[14] M. d. R. Diaz Zelada, G. J. Náder Abad y M. G. Román Holguín: 'Factores que influyen en el uso de una aplicación móvil financiera en cajas municipales de ahorro y crédito de la ciudad de Tacna'. Tesis de maestría, Perú: Neumann Business School, 2017

[15] S. Bayona-Oré y L. Leyva, «Factores Críticos de Aceptación de la Intención de Uso del Efectivo Móvil. Revista Ibérica de Sistemas e Tecnologias de Informação» vol. 1, n E28, pp. 972-980, 2020.

[16] J. Fernando Tavera y B. E. Londoño, «Factores Determinantes de la Aceptación Tecnológica del E-Commerce en Países Emergentes,» vol. 22, nº 31, pp. 101-119, 2012.

[17] R. Ferrer Cascales, A. Reig Ferrer, F. Pascual y N. Albaladejo Blázquez, «Evaluación de la satisfacción del uso de las redes sociales en formación,» Universidad de Alicante, vol. 1, $n^{\circ} 1$, pp. 1-14, 2012.

[18] F. Huamán-Delgado, C. Vílchez-Román y J. Briceño, «Aceptación Tecnológica de apps móviles para actividad física: un estudio basado en el modelo UTAUT2,»-Departamento Académico de Ciencias de la Gestión PUCP, vol. 1, n 1, pp. 1-20, 2017. 
[19] A. Cataldo, «Limitaciones y oportunidades del Modelo de Aceptación Tecnológica (TAM),» Universidad de Atacama, vol. 1, no 1, pp. 1-6, 2011

[20] M. Gutarra. Tesis validó el TAM y la Norma Técnica Peruana. Noticias. Universidad Nacional Mayor de San Marcos, 2013. Recuperado de http://www.unmsm.edu.pe/noticias/ver/2486

[21] F. Davis, «Perceived Usefulness, Perceived Ease of Use, and User Acceptance of Information Technology» MIS Quaterly, vol. 1, no 1, pp. 319-340, 1989.

[22] M. Fishbein y B. Ajzen, «attitude, intention, and behavior: an introduction to theory and research, Reading,» vol. $1, n^{\circ} 1$, pp. 1-20, 1975.

[23] F. Davis, «User acceptance of information technology: system characteristics, user perceptions and behavioral impacts.," vol. 1, n 38 , pp. 475-487, 1991.

[24] Rogers, E. (1995). Diffusion of innovation. New York: Free.

[25] R. Taylor y P. Todd, «Understanding Information Technology usage: A test of competing models,» Information Systems Research, vol. 6, n² 2, pp. 1-10, 1995a.

[26] Davis, F.D., Bagozzi, R.P., Warshaw, P.R., 1989. User acceptance of computer technology: A comparison of two theoretical models. Management Science 35, 982-1003.

[27] Yong Varela, Luis Antonio; Rivas Tovar, Luis Arturo; Chaparro, Julián;, «Modelo de aceptación tecnológica (TAM): un estudio de la influencia de la cultura nacional y del perfil del usuario en el uso de las TIC,» Innovar. Revista de Ciencias Administrativas y Sociales, vol. 20, nº 136, pp. 187-203, 2010.

[28] J. Matute Vallejo, Y. Polo Redondo y A. Utrillas Acerete, «Las características del boca-oído electrónico y su influencia en la intención de recompra online,» Revista Europea de Dirección y Economía de la Empresa, vol. 1, n² 24, pp. 61-75, 2015.

[29] Aguilera Serpa, C.: 'Impacto de los factores organizacionales y de usuarios en la aceptación y uso de software de gestión de proyecto en la mediana empresa en Lima'. Tesis de Maestría. Lima, Perú: Universidad Nacional Mayor de San Marcos, 2017.

[30] Alonso González, D.: 'Trabajo social y tecnología: aceptación y uso entre profesionales en formación'. Tesis de doctorado. Madrid, España: Universidad Complutense de Madrid, 2016.

[31] M. d. J. Araiza Vázquez y J. F. Ramírez Ramírez, «Factores que determinan la aceptación de la Plataforma Nexus por los estudiantes de la Escuela de Negocios de la UANL,» Daena: International Journal of Good Conscience, vol. 12, $\mathrm{n}^{\circ} 1$, pp. 200-233, 2017.

[32] C. J. Bravo, P. E. Ramírez y J. Arenas, «Aceptación de Reconocimiento Facial Como Medida de Vigilancia y Seguridad: Un Estudio Empírico en Chile,» Información Tecnológica, vol. 29, n² 2, pp. 115-122, 2017.

[33] J. C. Sánchez-Prieto, S. Olmos-Migueláñez y F. J. García-Peñalvo, «Motivación e innovación: Aceptación de tecnologías móviles en los maestros en formación.,» RIED, vol. 2, n 20 , pp. 273-292, 2017.

[34] M. E. Nizama Reyes, «Aceptación de los sistemas de información integrados y herramientas de la dirección regional de salud - Piura,» vol. 1, n² 2, pp. 59-66, 2014.

[35] C. Medina Molina, R. Rufin Moreno y M. Rey Moreno, «La adopción del e-Gobierno en entornos voluntarios,» vol. $1, n^{\circ}$ 19, pp. 42-52, 2013.-
[36] A. M. Reyes Huamán: 'Factores motivacionales de la aceptación de las redes sociales virtuales en las universidades del Perú'. Tesis de maestría, Lima: Universidad Nacional Mayor de San Marcos, 2015.

[37] J. A. Sánchez Torres y F.-J. Arroyo-Cañada, «Diferencias de la adopción del comercio electrónico entre países,» Elsevier, vol. 1, nº 7, pp. 141-150, 2016.

[38] J. F. Tavera y B. E. Londoño, «Factores determinantes de la aceptación tecnológica del e-commerce en países emergentes,» Revista Ciencias Estratégicas, vol. 22, n 31, pp. 101-119, 2014

[39] A. M. Villa Zapata, K. Ramírez Salazar y J. Tavera Mesías, «Antecedentes de la Intención de Uso de los sitios web de compras colectivas,» Escuela de Ingeniería de Antioquia, vol. 12, n 24, pp. 55-70, 2015.

[40] J. Cabero, B. Sampedro y O. M. Gallego. Valoraciones de la "Aceptación de la Tecnología de Formación Virtual" por profesores universitarios asistentes a un curso de formación virtual. EDUTEC Revista Electrónica de Tecnología Educativa, (56), 31-47. ISSN:1135- 9250, 2016.

[41] V. Venkatesh y H. Bala. Technology Acceptance Model 3 and a Research Agenda on Interventions. Decision Sciences, 39(2), 273-315, 2008.

[42] V. Venkatesh y F. Davis. A theoretical extension of the technology acceptance model: Four longitudinal field studies, (46):186-204, 2000

[43] Oficina Nacional de Procesos Electorales, «ONPE comparte exitoso Sistema de Gestión Documentaria con otras instituciones públicas,» 09 Noviembre 2018. [En línea]. Available: https://www.onpe.gob.pe/sala-prensa/notas-prensa/onpe-comparte-exitoso-sistema-gestion-documentaria-con-otras-instituciones-publicas/.

[44] I. Ajzen, «Attitudes, traits, and actions: Dispositional prediction of behavior in personality and social psychology,» Adv experimental social psychology, Volumen 20 , vol. $2, n^{\circ} 1$, pp. 11-39, 1987.

[45] F. Muñoz Leiva, La adopcion de una innovacion basada en la web (tesis doctoral), Granada: Universidad de Granada, 2008.

[46] J. R. Chavez Muñoz, E. A. Miranda Casas, N. J. Quispe Loyola y S. E. Robles Cossio, Factores que influyen en la intención de uso de tecnología de medios de pago móvil en negocios minoristas en Lima Metropolitana, Lima: Universidad ESAN, 2019.

[47] W. Schlesinger, A. Cervera y H. Calderón, «El papel de la confianza, la imagen y los valores compartidos en la creación de valor y lealtad: aplicación a la relación egresado-universidad,» Revista Española de Investigación de Marketing ESIC, vol. 1, no 18, pp. 126-139, 2014.

[48] M. d. J. Araiza Vásquez y E. Y. Pedraza Sánchez, Aceptación de servicios financieros en línea por los consumidores del área metropolitana de Monterrey, México: Universidad de Nuevo León, 2019.

[49] J. Henseler, C. Ringle y M. Sarstedt, «Testing measurement invariance of composites using partial least squares,» International Marketing Review, vol. 116, nº 1, pp. 2-20, 2016. 